$\mathbb{T}$ periodica polytechnica

Civil Engineering

$57 / 1(2013) 21+26$

doi: 10.3311/PPci.2138

http://periodicapolytechnica.org/ci

Creative Commons Attribution (1)

RESEARCH ARTICLE

\section{Numeric-Symbolic Solution for Satellite Trajectory Control by Pole Placement}

\author{
Béla Paláncz
}

Received 2012-01-30, revised 2012-05-07, accepted 2013-03-01

\begin{abstract}
Control design of satellites based on pole placement method results in determined or underdetermined multivariable polynomial systems. Since only the real solutions can be considered for hardware implementation, we are looking for exclusively these solutions. In this study we suggest a numeric-symbolic approach to compute only the real solutions directly. Employing computer algebra (Dixon or reduced Gröbner basis) a condition can be formulated for the free variables as parameters of the underdetermined system, which ensures only real solutions. Numerical example illustrates the procedure and the effectivity of the control law.
\end{abstract}

\section{Keywords}

satellite control p pole placement $\cdot$ symbolic-numeric computation $\cdot$ multivariable polynomial system $\cdot$ Dixon resultant $\cdot r e-$ duced Gröbner basis

\section{Béla Paláncz}

Budapest University of Technology and Economics, Department of Photogrammetry and Geoinformatics, 1521 Budapest, Hungary

e-mail:palancz@epito.bme.hu

\section{Introduction}

Geodesic satellites play an important role in navigation (Global Navigation Satellite System, GNSS), in environmental monitoring like global warming, desertification, flooding, space born meteorology (US based Geostationary Operational Environmental Satellite, GEOS, Europe owned METEorological SATellite, METEOSAT) as well as in positioning of any point on Earth from space, preliminary designed to be used by the US military (Global Positioning System, GPS), see Awange et al. (2010) [1] as well as Somodi and Földváry (2011) [16].

The control of the trajectories of such satellites in order to ensure their proper and reliable operation is an extremely important task. Attitude control (angular orientation) keeping the satellite pointed in the right direction as well as orbit control are needed so, that the optical system covers the programmed ground area at all times. However, the satellite tends to change its orientation due to torque produced by environment (drag of residual atmosphere on solar array, solar radiation pressure, the gravitational acceleration of the sun and moon and the effect of the earth not being spherical, etc.) or by itself (due to movement of mechanical parts, etc). Thus the position and motion of the satellite are continuously controlled by a programmed control loop consisting of sensors (like rate gyros) measuring the satellite's attitude. The on board computer processes these measurements and generates commands according to the control law of the controller. These commands are carried out by the actuators (like micro-trusters) to ensure correct pointing and orbit. A detailed description of the design and evaluation such control systems can be found in Paluszek at al (2009) [13].

In this study the design of a control law via numeric - symbolic techniques is suggested. For control design one basically needs the dynamical model of the satellite motion. Surprisingly, relatively simple model can be satisfactory for such task, see Neokleous (2007) [10].

Now we consider a simple but realistic dynamical model of the satellite, see Kailath (1980) [7]. Generally polar coordinates are used for the satellite being in a circular, equatorial orbit. The goal of the feedback is to keep the satellite in the same orbit when disturbances such as aerodynamic drag cause it to deviate. 
The state vector is $\mathbf{x}=[r \dot{\mathbf{r}} \theta \dot{\theta}]$ with $r$ and $\theta$ the deviations from the reference orbit and the reference attitude, respectively and the input is $\mathbf{u}=\left[u_{r}, u_{t}\right]$, with $u_{r}$ and $u_{t}$ respectively the radial and tangential thrusters. The linearized state-space equations around the reference orbit are represented by the following matrices,

$$
A=\left(\begin{array}{cccc}
0 & 1 & 0 & 0 \\
3 \omega_{0}^{2} & 0 & 0 & 2 \omega_{0} r_{0} \\
0 & 0 & 0 & 1 \\
0 & -2 \frac{\omega_{0}}{r_{0}} & 0 & 0
\end{array}\right) \text { and } B=\left(\begin{array}{cc}
0 & 0 \\
\frac{1}{v} & 0 \\
0 & 0 \\
0 & \frac{1}{v r_{0}}
\end{array}\right)
$$

where the radius of the orbit $r_{0}$, angular velocity $\omega_{0}$ and $v$ is the mass of the satellite. Supposing that the satellite completely controllable with tangential thruster, Dorf and Bishop (1998) [5], let

$$
C=\left(\begin{array}{llll}
0 & 0 & 1 & 0 \\
0 & 0 & 0 & 1
\end{array}\right)
$$

There are numerous control design techniques developed for linear dynamic systems. Here we consider the pole placement technique, Byrnes (1989) [2]. The advantages of this approach are the simplicity and the robustness, both are very important in case of satellite control.

\section{Pole placement problem}

Assuming, we are given a linear system with $m$ inputs $u \in \mathbb{R}^{m}$, $p$ outputs $\mathbf{y} \in \mathbb{R}^{p}$ by three matrices: $A \in \mathbb{R}^{n \times n}, B \in \mathbb{R}^{n \times m}$ and $C \in \mathbb{R}^{p \times n}$, where $n$ equals the number of internal states stored by the vector $\mathbf{x} \in\left(\mathbb{R}^{n}\right.$. These three matrices define the system of linear first order differential equations:

$$
\begin{gathered}
\dot{\mathbf{x}}(t)=A \mathbf{x}(t)+B \mathbf{u}(t) \\
\dot{\mathbf{y}}(t)=C \mathbf{x}(t) .
\end{gathered}
$$

Our task is to find a control law,

$$
\mathbf{u}_{c}(t)=K(t) y(t)
$$

which provides the system input to stabilize the system,

$$
\mathbf{u}(t)=\mathbf{r}(t)-\mathbf{u}_{c}(t)
$$

where $\mathbf{r}$ is a reference input vector, see Fig. 1.

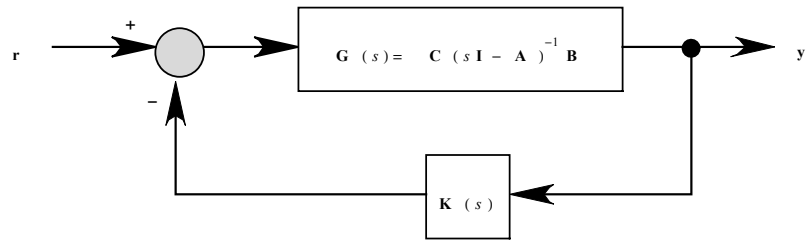

Fig. 1. System with output-feedback pole assignment compensator in frequency-domain representation

The control law can be represented by a linear system, by tuples of four matrices $(F, L, H, M)$

$$
\mathbf{u}_{c}(t)=H \mathbf{z}(t)+M \mathbf{y}(t)
$$

$$
\dot{\mathbf{z}}(t)=F \mathbf{z}(t)+L \mathbf{y}(t)
$$

where $\mathbf{z} \in \mathbb{R}^{q}$. The compensator which realizes this control law is called as $q$ th-order dynamic compensator. The most simple realization $(q=0)$ is a single constant matrix $M \in \mathbb{C}^{m \times p}$, which called as a static compensator,

$$
\mathbf{u}_{c}(t)=M \mathbf{y}(t)
$$

There are many different methods to solve this linear control problem. One of the classical and most simple methods is the pole placement technique. In this case the compensator realizes a feedback law, which ensures the prespecified closed-loop system's poles, $\lambda_{1}, \lambda_{2}, \ldots, \lambda_{n+q}$.

It means that if the system matrices $(A, B, C)$ are known and the assigned poles $\left(\lambda_{i}\right.$ 's are given, then the state space matrices of the compensator $(F, L, H, M)$ should be computed.

You can find many iterative numerical approaches to implement this technique like Ackermann's formula employing controllability matrix and the characteristic polynomial of matrix $A$, robust pole assignment method using iteration, recursive algorithm using Hessenberg-form, explicit and implicit $Q R$ algorithm employing QR decomposition and Schur method with Schur decomposition, see Datta et al (2003) [3].

These methods are built in the control design packages of leading computing systems like Mathematica and MATLAB. However, most of them suffer from numerical instability resulted by ill-conditioned linear system and in case of multiplied poles they essentially fail because of singularity, Rosenthal and Willems (1998) [15].

Pole placement problem leads to a system of multivariate polynomial system, which can have real as well as complex solutions, although we are interested in only the real solutions, since only these can be practically implemented in the hardware elements of the control loop. To find all of the real solutions exclusively is not an easy task, see Dickenstein and Emiris (2005) [15].

Recently, numerical homotopy method is suggested as a symbolic-numeric solution of the problem using Pieri homotopy and implemented in software combining MATLAB and Maple based PHCpack, see Verschelde and Wang (2004) [17]. This method computes all of the solutions of the system, then the real ones can be selected.

In this study we introduce an alternative approach, which makes it possible to compute only the real solutions, directly. To illustrate this method, here we consider two basic approaches for developing the multivariate polynomial system to be solved.

\section{Transfer function approach}

In this case we use the denominator of the closed loop transfer function directly. Employing the Laplace transform of Eq. (5) and (6), the transfer function of the closed loop can be expressed 
as,

$$
Q(s)=\frac{\mathcal{L}(y)}{\mathcal{L}(r)}=\left(I_{p}+G(s) K(s)\right)^{-1} G(s)
$$

where $G(s)$ is the transfer function of the linear system (open -loop), with the Laplace transform of Eqs. (3) and (4) we get,

$$
G(s)=\frac{\mathcal{L}(y)}{\mathcal{L}(u)}=C\left(s I_{n}-A\right)^{-1} B
$$

In case of MIMO system (multiple input- multiple output) $Q(s)$ is a matrix with polynomial entries. These $\lambda_{i}$ values should be the roots of the denominators of the elements of $Q(s)$. Since all of the elements have the same denominator, $Q(s)$ can be expressed as,

$$
Q(s)=\frac{N(s)}{P(s)}
$$

Consequently, we get the following polynomial system,

$$
P\left(\lambda_{i}\right)=0, \quad i=1,2, \ldots, n+q
$$

Designing compensator means to compute the elements of the tuples of four matrices $(F, L, H, M)$ under this condition. In general, we can set $n+q$ poles as condition, and we have $(m+q)$ $(p+q)$ matrix elements as unknowns, see Fig.2

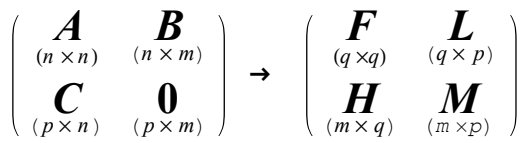

a)

b)

Fig. 2. State space matrices of the control system a) system, b) compensator

Now, in the system Eq.(13), the elements of $(F, L, H, M)$ are unknown variables, while the elements $A, B, C$ are known and the $\lambda_{i}$ values are given. Therefore Eq. (13) can be considered as a multivariate polynomial in terms of the unknown elements of $(F, L, H, M)$.

However there is another way to compute the tuples of the four matrices $(F, L, H, M)$, namely from the characteristic equation of the closed loop.

\section{Characteristic polynomial approach}

Let us suppose that $(A, B, C)$ are real matrices, then exist matrices $(F, L, H, M)$, and the following polynomial of degree $n \times q$ which is called as the characteristic polynomial of the closed loop with negative feedback,

$$
\phi(\lambda)=\operatorname{det} \Omega
$$

where $\Omega$ is a square matrix of $(n+q) \times(n+q)$,

$$
\Omega=\left(\begin{array}{cc}
\lambda I_{n}-A+B M C & B H \\
-L C & \lambda I_{q}-F
\end{array}\right)
$$

All of the poles of the closed loop satisfy this polynomial, namely

$$
\phi\left(\lambda_{i}\right)=0, \quad i=1,2, \ldots, n+q
$$

\section{Computation of static compensator}

In our case $n=4, m=2$ and $p=2$. In addition $q=0$ and $m \times p=n$ therefore the polynomial system, Eq. (13) is linear, see Wang (1996) [18]. However this system is frequently ill-conditioned and in case of multiplied poles is singular. The transfer function of the system is, see Eq. (11)

$$
G(s)=C\left(s I_{n}-A\right)^{-1} B=\left(\begin{array}{cc}
-\frac{2 s \omega_{0}}{v r_{0}\left(s^{4}+s^{2} \omega_{0}^{2}\right)} & \frac{s^{2}-3 \omega_{0}^{2}}{v r_{0}\left(s^{4}+s^{2} \omega_{0}^{2}\right)} \\
-\frac{2 s^{2} \omega_{0}}{v r_{0}\left(s^{4}+s^{2} \omega_{0}^{2}\right)} & \frac{s^{3}-3 s \omega_{0}^{2}}{v r_{0}\left(s^{4}+s^{2} \omega_{0}^{2}\right)}
\end{array}\right)
$$

The elements of the transfer function of the closed loop system, see Eq.(10)

$$
Q(s)=\left(I_{p}+G(s) K(s)\right)^{-1} G(s)=\frac{1}{P(s)}\left(\begin{array}{cc}
2 s \omega_{0} & s^{2}-3 \omega_{0}^{2} \\
2 s^{2} \omega_{0} & s^{3}-3 s \omega_{0}^{2}
\end{array}\right)
$$

where

$$
\begin{aligned}
P(s)= & s^{4} v r_{0}+s^{2} v r_{0} \omega_{0}^{2}-2 s \omega_{0} m_{1,1}-2 s^{2} \omega_{0} m_{1,2}+s^{2} m_{2,1} \\
& -3 \omega_{0}^{2} m_{2,1}+s^{3} m_{2,2}-3 s \omega_{0}^{2} m_{2,2}
\end{aligned}
$$

It goes without saying that the characteristic polynomial approach leads to the same polynomial. Now Eq. (14) reduces to

$$
\phi(\lambda)=\operatorname{det}\left(\lambda I_{n}-A+B M C\right)
$$

therefore

$$
\begin{aligned}
\phi(\lambda)= & \lambda^{4}+\lambda^{2} \omega_{0}^{2}-\frac{2 \lambda \omega_{0} m_{1,1}}{v r_{0}}-\frac{2 \lambda^{2} \omega_{0} m_{1,2}}{v r_{0}} \\
& +\frac{\lambda^{2} m_{2,1}}{v r_{0}}-\frac{3 \omega_{0}^{2} m_{2,1}}{v r_{0}}+\frac{\lambda^{3} m_{2,2}}{v r_{0}} \\
& -\frac{3 \lambda \omega_{0}^{2} m_{2,2}}{v r_{0}} \omega_{0}^{2} m_{2,1}+s^{3} m_{2,2}-3 s \omega_{0}^{2} m_{2,2}
\end{aligned}
$$

Since $v r_{0} \neq 0$, Eq. (17) and Eq. (19) have the same roots.

Now, considering Eq. (9) the compensator gain is $K(s) \equiv M$ constant matrix, with $m \times p=2 \times 2$ entries,

$$
M=\left(\begin{array}{ll}
m_{1,1} & m_{1,2} \\
m_{2,1} & m_{2,2}
\end{array}\right)
$$

We can assign $n+q=4+0=4$ poles. Let the desired poles are $\left\{\lambda_{1}, \lambda_{2}, \lambda_{3}, \lambda_{4}\right\}$. All prespecified $\lambda_{i}$ should be the root of the polynomial $P$ (or $\phi$ ). Our problem has no free parameters, since $(n+q)=4$ is equal to $(m+q)(p+q)=(2+0) \times(2+0)=4$, consequently the linear system for $m_{i, j}$ is determined,

$$
\begin{aligned}
& v r_{0} \lambda_{1}^{4}+v r_{0} \lambda_{1}^{2} \omega_{0}^{2}-2 \lambda_{1} \omega_{0} m_{1,1}-2 \lambda_{1}^{2} \omega_{0} m_{1,2}+\lambda_{1}^{2} m_{2,1} \\
& -3 \omega_{0}^{2} m_{2,1}+\lambda_{1}^{3} m_{2,2}-3 \lambda_{1} \omega_{0}^{2} m_{2,2}=0 \\
& v r_{0} \lambda_{2}^{4}+v r_{0} \lambda_{2}^{2} \omega_{0}^{2}-2 \lambda_{2} \omega_{0} m_{1,1}-2 \lambda_{2}^{2} \omega_{0} m_{1,2}+\lambda_{2}^{2} m_{2,1} \\
& -3 \omega_{0}^{2} m_{2,1}+\lambda_{2}^{3} m_{2,2}-3 \lambda_{2} \omega_{0}^{2} m_{2,2}=0 \\
& v r_{0} \lambda_{3}^{4}+v r_{0} \lambda_{3}^{2} \omega_{0}^{2}-2 \lambda_{3} \omega_{0} m_{1,1}-2 \lambda_{3}^{2} \omega_{0} m_{1,2}+\lambda_{3}^{2} m_{2,1} \\
& -3 \omega_{0}^{2} m_{2,1}+\lambda_{3}^{3} m_{2,2}-3 \lambda_{3} \omega_{0}^{2} m_{2,2}=0 \\
& v r_{0} \lambda_{4}^{4}+v r_{0} \lambda_{4}^{2} \omega_{0}^{2}-2 \lambda_{4} \omega_{0} m_{1,1}-2 \lambda_{4}^{2} \omega_{0} m_{1,2}+\lambda_{4}^{2} m_{2,1} \\
& -3 \omega_{0}^{2} m_{2,1}+\lambda_{4}^{3} m_{2,2}-3 \lambda_{4} \omega_{0}^{2} m_{2,2}=0
\end{aligned}
$$


The solution can be expressed via Dixon resultant or reduced Gröbner basis,

$$
\begin{aligned}
m_{1,1}= & \frac{v r_{0}}{2 \omega_{0}}\left(\lambda_{1} \lambda_{2} \lambda_{3}+\lambda_{1} \lambda_{2} \lambda_{4}+\lambda_{1} \lambda_{3} \lambda_{4}+\lambda_{2} \lambda_{3} \lambda_{4}+3 \lambda_{1} \omega_{0}^{2}\right. \\
& \left.+3 \lambda_{2} \omega_{0}^{2}+3 \lambda_{3} \omega_{0}^{2}+3 \lambda_{4} \omega_{0}^{2}\right) \\
m_{1,2}= & -\frac{1}{6 \omega_{0}^{3}} v r_{0}\left(\lambda_{1} \lambda_{2} \lambda_{3} \lambda_{4}+3 \lambda_{1} \lambda_{2} \omega_{0}^{2}+3 \lambda_{1} \lambda_{3} \omega_{0}^{2}\right. \\
& \left.+3 \lambda_{2} \lambda_{3} \omega_{0}^{2}+3 \lambda_{1} \lambda_{4} \omega_{0}^{2}+3 \lambda_{2} \lambda_{4} \omega_{0}^{2}+3 \lambda_{3} \lambda_{4} \omega_{0}^{2}-3 \omega_{0}^{4}\right) \\
m_{2,1}= & -\frac{v r_{0} \lambda_{1} \lambda_{2} \lambda_{3} \lambda_{4}}{3 \omega_{0}^{2}} \\
m_{2,2}= & -v r_{0}\left(\lambda_{1}+\lambda_{2}+\lambda_{3}+\lambda_{4}\right)
\end{aligned}
$$

\section{6. Computation of a dynamic compensator}

Considering the Laplace transform of Eq. (8),

$$
s \mathcal{L}(z)=F \mathcal{L}(z)+\mathcal{L}(y)
$$

Then

$$
\mathcal{L}(z)\left(s I_{q}-F\right)=L \mathcal{L}(y)
$$

Expressing $\mathcal{L}(z)$ and substituting it into the Laplace transform of Eq. (7), we obtain,

$$
\mathcal{L}\left(u_{c}\right)=H\left(s I_{q}-F\right)^{-1} L \mathcal{L}(y)+M \mathcal{L}(y)
$$

Therefore the transfer function of the dynamical compensator, is,

$$
K(s)=\frac{\mathcal{L}\left(u_{c}\right)}{\mathcal{L}(y)}=H\left(s I_{q}-F\right)^{-1} L+M
$$

Let $q=1$, then $n+q=4+1=5$ poles to be assigned, $\left\{\lambda_{1}, \lambda_{2}, \lambda_{3}, \lambda_{4}, \lambda_{5}\right\}$, and there are $(m+q)(p+q)=(2+1) \times(2+1)=$ 9 parameters to be computed. The state matrices of the dynamic compensator are, see Fig. 2,

$$
\begin{array}{ll}
F=\left(f_{1,1}\right), & L=\left(\begin{array}{ll}
l_{1,1} & l_{1,2}
\end{array}\right), \\
H=\left(\begin{array}{l}
h_{1,1} \\
h_{2,1}
\end{array}\right), & M=\left(\begin{array}{ll}
m_{1,1} & m_{1,2} \\
m_{2,1} & m_{2,2}
\end{array}\right)
\end{array}
$$

Consequently we have 4 free parameters. Let us compute the transfer function of the compensator, see Eq. (24),

$$
K=H\left(s I_{q}-F\right)^{-1} L+M=\left(\begin{array}{ll}
\frac{h_{1,1} l_{1,1}}{s-f_{1,1}}+m_{1,1} & \frac{h_{1,1} l_{1,2}}{s-f_{1,1}}+m_{1,2} \\
\frac{h_{2,1} l_{1,1}}{s-f_{1,1}}+m_{2,1} & \frac{h_{2,1} l_{1,2}}{s-f_{1,1}}+m_{2,2}
\end{array}\right)
$$

The transfer function of the closed loop is, see Eq. (10),

$$
Q=\frac{\left(s-f_{1,1}\right)}{P(s)}\left(\begin{array}{cc}
-2 s \omega_{0} & \left(s^{2}-3 \omega_{0}^{2}\right) \\
-2 s^{2} \omega_{0} & s\left(s^{2}-3 \omega_{0}^{2}\right)
\end{array}\right)
$$

where,

$$
\begin{aligned}
P(s)= & s^{5} v r_{0}+s^{3} v r_{0} \omega_{0}^{2}-s^{4} v r_{0} f_{1,1}-s^{2} v r_{0} \omega_{0}^{2} f_{1,1}-2 s \omega_{0} h_{1,1} l_{1,1} \\
& +s^{2} h_{2,1} l_{1,1}-3 \omega_{0}^{2} h_{2,1} l_{1,1}-2 s^{2} \omega_{0} h_{1,1} l_{1,2}+s^{3} h_{2,1} l_{1,2} \\
& -3 s \omega_{0}^{2} h_{2,1} l_{1,2}-2 s^{2} \omega_{0} m_{1,1}+2 s \omega_{0} f_{1,1} m_{1,1}-2 s^{3} \omega_{0} m_{1,2} \\
& +2 s^{2} \omega_{0} f_{1,1} m_{1,2}+s^{3} m_{2,1}-3 s \omega_{0}^{2} m_{2,1}-s^{2} f_{1,1} m_{2,1} \\
& +3 \omega_{0}^{2} f_{1,1} m_{2,1}+s^{4} m_{2,2}-3 s^{2} \omega_{0}^{2} m_{2,2}-s^{3} f_{1,1} m_{2,2}+3 s \omega_{0}^{2} f
\end{aligned}
$$

As the alternative method, let us employ again the characteristic polynomial approach,

$$
\begin{aligned}
\Omega & =\left(\begin{array}{ccc}
\lambda I_{4}-A+B M C & B H \\
-L C & \lambda I_{1}-F
\end{array}\right) \\
& =\left(\begin{array}{ccccc}
\lambda & -1 & 0 & 0 & 0 \\
-3 \omega_{0}^{2} & \lambda & \frac{m_{1,1}}{v} & -2 r_{0} \omega_{0}+\frac{m_{1,2}}{v} & \frac{h_{1,1}}{v} \\
0 & 0 & \lambda & -1 & 0 \\
0 & \frac{2 \omega_{0}}{r_{0}} & \frac{m_{2,1}}{v r_{0}} & \lambda+\frac{m_{2,2}}{v r_{0}} & \frac{h_{2,1}}{v r_{0}} \\
0 & 0 & -l_{1,1} & -l_{1,2} & \lambda-f_{1,1}
\end{array}\right)
\end{aligned}
$$

then again the roots of the polynomials $P(s)$ and $\phi(\lambda)=\operatorname{det}(\Omega)$ are the same. Now, we have 5 poles to be assigned and all of them should satisfy this polynomial. Consequently there are a polynomial system of five equations to be solved for the coefficients of matrices $F, L, H$ and $M$,

$$
P\left(\lambda_{i}\right)=0, \quad i=1,2, \ldots, 5
$$

The number of unknowns is 9, see Eq. (25). Therefore 4 of them can be considered as free parameters. For example, let us choose $f_{1,1}, l_{1,1}, h_{1,1}, m_{1,1}$ and $m_{2,2}$ as unknowns and $l_{1,2}, h_{2,1}, m_{1,2}$ and $m_{2,1}$ as parameters. Now applying Dixon resultant, to the system Eq. (27), we get the following polynomial for $f_{1,1}$,

$$
\alpha f_{1,1}^{3}+\beta f_{1,1}^{2}+\gamma f_{1,1}+\delta=0
$$

where

$$
\begin{aligned}
& \alpha=-12 v r_{0}\left(\lambda_{1}-\lambda_{2}\right)\left(\lambda_{1}-\lambda_{3}\right)\left(\lambda_{2}-\lambda_{3}\right)\left(\lambda_{1}-\lambda_{4}\right)\left(\lambda_{2}-\lambda_{4}\right) \\
& \cdot\left(\lambda_{3}-\lambda_{4}\right)\left(\lambda_{1}-\lambda_{5}\right)\left(\lambda_{2}-\lambda_{5}\right)\left(\lambda_{3}-\lambda_{5}\right)\left(\lambda_{4}-\lambda_{5}\right) \omega_{0}^{4} h_{2,1} l_{1,2} \\
& \beta=-12\left(\lambda_{1}-\lambda_{2}\right)\left(\lambda_{1}-\lambda_{3}\right)\left(\lambda_{2}-\lambda_{3}\right)\left(\lambda_{1}-\lambda_{4}\right)\left(\lambda_{2}-\lambda_{4}\right) \\
& \quad \cdot\left(\lambda_{3}-\lambda_{4}\right)\left(\lambda_{1}-\lambda_{5}\right)\left(\lambda_{2}-\lambda_{5}\right)\left(\lambda_{3}-\lambda_{5}\right)\left(\lambda_{4}-\lambda_{5}\right) \\
& \quad \cdot\left(-v r_{0} \lambda_{1}-v r_{0} \lambda_{2}-v r_{0} \lambda_{3}-v r_{0} \lambda_{4}-v r_{0} \lambda_{5}\right) \omega_{0}^{4} h_{2,1} l_{1,2} \\
& \gamma=-12\left(\lambda_{1}-\lambda_{2}\right)\left(\lambda_{1}-\lambda_{3}\right)\left(\lambda_{2}-\lambda_{3}\right)\left(\lambda_{1}-\lambda_{4}\right)\left(\lambda_{2}-\lambda_{4}\right) \\
& \cdot\left(\lambda_{3}-\lambda_{4}\right)\left(\lambda_{1}-\lambda_{5}\right)\left(\lambda_{2}-\lambda_{5}\right)\left(\lambda_{3}-\lambda_{5}\right)\left(\lambda_{4}-\lambda_{5}\right) \\
& \cdot \omega_{0}^{4} h_{2,1} l_{1,2}\left(v r _ { 0 } \left(\lambda_{3} \lambda_{4}+\lambda_{3} \lambda_{5}+\lambda_{4} \lambda_{5}+\lambda_{2}\left(\lambda_{3}+\lambda_{4}+\lambda_{5}\right)\right.\right. \\
& \left.\left.\quad+\lambda_{1}\left(\lambda_{2}+\lambda_{3}+\lambda_{4}+\lambda_{5}\right)-\omega_{0}^{2}\right)-h_{2,1} l_{1,2}+2 \omega_{0} m_{1,2}-m_{2,1}\right)
\end{aligned}
$$

and

$$
\delta=0
$$

Since $q=1$, therefore $f_{1,1} \neq 0$ and the polynomial can be reduced to a polynomial of second order,

$$
\alpha f_{1,1}^{2}+\beta f_{1,1}+\gamma=0
$$

In practice, the compensators can realize only real feedback law. If the matrices $(A, B, C)$ are real and the condition $q(m+p)+$ $m p>n+q$ is true, then there exist real matrices $(F, L, H, M)$, see Rosenthal and Wang (1996) [14]. Since in our case $1 \times(2+$ 2) $+2 \times 2=8>4+1=5$, we are looking for only real solutions. Then the following constrain has to be valid for the parameters, 
Tab. 1. Numerical solution of the pole placement problem

\begin{tabular}{ccccc}
\hline$f_{1,1}$ & $l_{1,1}$ & $h_{1,1}$ & $m_{1,1}$ & $m_{2,2}$ \\
\hline-7.80999 & -473.116 & -11.0544 & -719.883 & 8.26297 \\
\hline-8.97886 & -503.506 & -12.0482 & -719.315 & 7.18729 \\
\hline
\end{tabular}

$$
c=\beta^{2}-4 \alpha \gamma \geq 0
$$

Considering Eq. (28),

$$
\begin{aligned}
& c=144 v r_{0}\left(\lambda_{1}-\lambda_{2}\right)^{2}\left(\lambda_{1}-\lambda_{3}\right)^{2}\left(\lambda_{2}-\lambda_{3}\right)^{2}\left(\lambda_{1}-\lambda_{4}\right)^{2} \\
& \cdot\left(\lambda_{2}-\lambda_{4}\right)^{2}\left(\lambda_{3}-\lambda_{4}\right)^{2}\left(\lambda_{1}-\lambda_{5}\right)^{2}\left(\lambda_{2}-\lambda_{5}\right)^{2}\left(\lambda_{3}-\lambda_{5}\right)^{2} \\
& \cdot\left(\lambda_{4}-\lambda_{5}\right)^{2} \omega_{0}^{8} h_{2,1}^{2} l_{1,2}^{2}\left(v r_{0}\left(\lambda_{1}+\lambda_{2}+\lambda_{3}+\lambda_{4}+\lambda_{5}\right)^{2}\right. \\
& -4\left(v r _ { 0 } \left(\lambda_{3} \lambda_{4}+\lambda_{3} \lambda_{5}+\lambda_{4} \lambda_{5}+\lambda_{2}\left(\lambda_{3}+\lambda_{4}+\lambda_{5}\right)\right.\right. \\
& \left.\left.\left.+\lambda_{1}\left(\lambda_{2}+\lambda_{3}+\lambda_{4}+\lambda_{5}\right)-\omega_{0}^{2}\right)-h_{2,1} l_{1,2}+2 \omega_{0} m_{1,2}-m_{2,1}\right)\right) \geq 0
\end{aligned}
$$

A more detailed mathematical analysis of the solutions of this polynomial system can be found in Paláncz (2013) [12].

\section{Numerical example for the dynamic compensator}

Now, $q=1$, therefore we need $n+q=4+1=5$ poles to be assigned. The values of poles and the model parameters are from Verschelde and Wang (2004) [17],

$$
\lambda_{1} \rightarrow \frac{-2+i}{\sqrt{5}}, \lambda_{2} \rightarrow \frac{-2-i}{\sqrt{5}}, \lambda_{3} \rightarrow-5, \lambda_{4} \rightarrow-7, \lambda_{5} \rightarrow-3
$$

and

$$
v=0.74564, \quad \omega_{0}=0.345354, \quad r_{0}=1.2342
$$

Let us select the free parameters as

$$
h_{2,1}=1, l_{1,2}=1, m_{1,2}=1 \text {, }
$$

\subsection{Numerical results}

Considering Eq. (29)

$$
m_{2,1} \geq 25.6857
$$

We choose $m_{2,1}=26$. Now substituting these numerical data into Eq. (27), it leads to a polynomial system for the unknown matrix coefficients $f_{1,1}, l_{1,1}, h_{1,1}, m_{1,1}, m_{2,2}$. This system can be easily solved by the numeric polynomial solver of Mathematica, NSolve based on numerical Groebner basis. The condition Eq.(30) ensures real solutions, namely there are two of them, see Table 1.

Considering strictly equal relation in Eq. (30) the two solutions will be the same. To check our solutions the eigenvalues of the state space form of the closed loop, see Eq. (15),

$$
S=\left(\begin{array}{cc}
A-B M C & -B H \\
L C & F
\end{array}\right)
$$

can be computed. The eigenvalues of $S$ are the same as the assigned values of $\lambda_{i}$ 's.
7.2 Simulation of the dynamic performance of the control system

In order to compare the dynamic behavior of the system with and without control, we simulate its dynamic response for a unitstep disturbance taking one second. Fig.3 shows the performance of open loop without compensator,

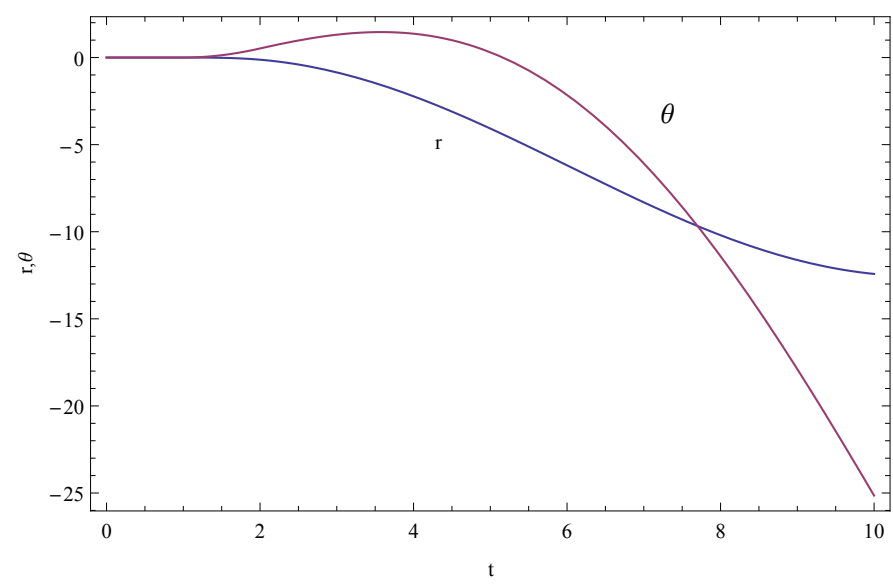

Fig. 3. System response without compensator

Applying compensator based on the first solution in Table 1, Fig. 4 shows the performance of the controlled system with this first order dynamic compensator,

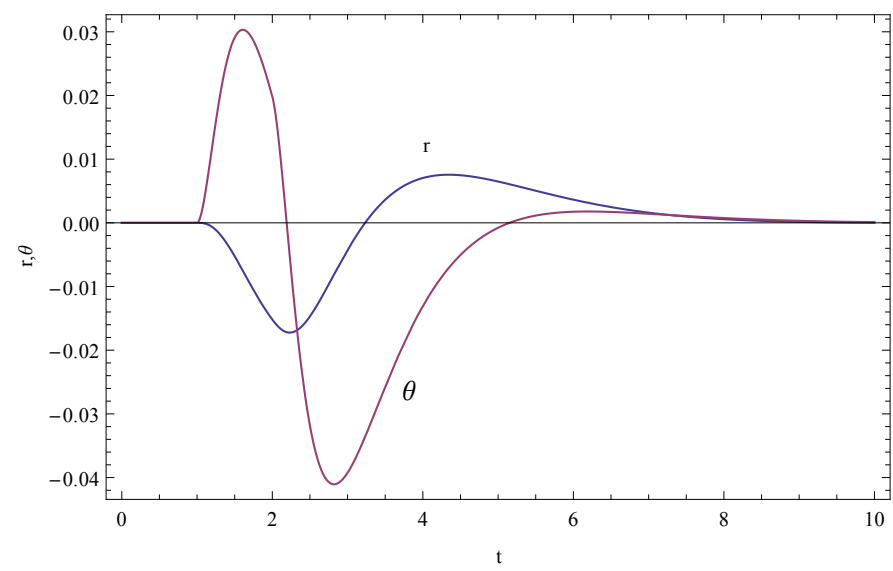

Fig. 4. System response with dynamic compensator

The symbolic and numeric computations were carried out with Mathematica. The Dixon resultant package developed and implemented by Nakos and Williams (1997) [8] and (2002) [9] was employed. To simulate the dynamical performance of the controlled satellite system the Control Application package of Mathematica was used, see Paláncz et al. (2005) [11].

\section{Conclusions}

In this contribution the application of computer algebra to determine pole-placement control law for controlling satellite trajectory was demonstrated. Employing Dixon resultant or reduced Groebner basis the matrices of the static controller can be computed in symbolic form. In case of dynamic controller, a constrain ensuring only real solutions of the multivariate polynomial system can be given. Consequently, the proper selection 
of the free parameters of the controller matrices provides the real solutions directly, without computing all solutions of the system numerically. Example illustrates, that this type of solution of the control law improves the dynamic performance of the satellite system effectively. Further improvement of this suggested method can be carried out via utilization of the non-uniqueness of the pole placement solution, namely defining the values of the free parameters in optimal way using the minimum possible fuel consumption via hypothetical loop-decoupling, see Juang (1997) [6].

\section{References}

1 Awange J, Grafarend E, Paláncz B, Zaletnyik P, Algebraic Geodesy and Geoinformatics, Springer; Berlin, 2010, DOI 10.1007/978-3-642-12124-1.

2 Byrnes C, Pole assignment by output feedback, In: Nijmacher H, Schumacher $\mathbf{J}$ (eds.), Three Decades of Mathematical Systems Theory, Springer; Berlin, 1989.

3 Datta B, Sarkissian D, Bakshee I, Advanced Numerical Methods, Wolfram Research, Inc.; Champaign, USA, 2003.

4 Dickenstein A, Emiris I, Solving Polynomial Equations, Foundations, Algorithms and Applications, Springer; Berlin.

5 Dorf GH, Bishop RH, Modern Control Systems, Addison-Wesley; New York, 1998.

6 Juang J, On parametric pole-placement for a class of linear systems, J. of Marine Sci. Techn, 5(1), (1997), 1-12.

7 Kailath T, Linear Systems, Prentice - Hall, Inc.; Englewood Cliffs, N.J., 1980.

8 Nakos G, Williams R, Elimination with the Dixon resultant, Mathematica in Education and Research, 6(1), (1997), 11-22.

9 Nakos G, Williams R, A fast algorithm implemented in Mathematica provides one-step elimination of a block of unknowns from a system of polynomial equations, 2002, http://library.wolfram.com/infocenter/ MathSource/2597/ MathSource.

10 Neokleous K, Modeling and control of a satellite's geostationary orbit, Master thesis, Lulea Uni. of Techn. Uni., Prague, 2007.

11 Paláncz B, Benyo Z, Kovács L, Control System Professional Suite, IEEE Control System Magazine, 25(2), (2005), 67-75, DOI 10.1109/MCS.2005.1411385.

12 Paláncz B, Application of Dixon resultant to satellite trajectory control by pole placement, J. of Symbolic Computation, 50(2), (2013), 79-99, DOI 10.1016/j.jsc.2012.05.007.

13 Paluszek M, Bhatta P, Griesemer P, Mueller J, Thomas S, Spacecraft Attitude and Orbit Control, Princeton Satellite Systems, Inc.; Plainsboro N.J., 2009.

14 Rosenthal J, Wang X, Output Feedback Pole Placement with Dynamic Compensator, IEEE Trans. On Automatic Control, 50(2), (1996), 830-842, DOI 10.1109/9.506235.

15 Rosenthal J, Willems $\mathbf{J}$, Open problems in the area of pole placement, In: Blondel V, Sontag E, Vidyasagar M, Willems J (eds.), Open Problems in Mathematical Systems and Control Theory, Springer; Berlin, 1998.

16 Somodi B, Földváry L, Application of Numerical Integration Techniques for Orbit Determination of State-of-the-art LEO Satellites, Periodica Polytechnica-Civil Engineering, 55(2), (2011), 99-106, DOI 10.3311/pp.ci.2011-2.02.

17 Verschelde J, Wang Y, Computing Dynamic Output Feedback Laws, IEEE Trans. On Automatic Control, 49(8), (2004), 1393-1397, DOI 10.1109/TAC.2004.832680.

18 Wang X, Grassmannian, Central Projection, and Output Feedback Pole Assignment of Linear Systems, IEEE Trans. On Automatic Control, 41(6), (1996), 786-794, DOI 10.1109/9.506231. 